\title{
Percepción sobre el apoyo social recibido tras el diagnóstico de cáncer de mama: La experiencia dos años después del diagnóstico
}

\author{
Elena Torralba-Martínez ${ }^{1}$ y Núria Codern-Bové ${ }^{2}$ \\ 1Universitat Autònoma de Barcelona, España | elenatm4@gmail.com \\ https://orcid.org/0000-0001-5716-0898 \\ ${ }^{2}$ Escola Universitària d'Infermeria i Teràpia Ocupacional de Terrassa (EUIT), \\ Universitat Autònoma de Barcelona, Terrassa, España | \\ nuriacodern@euit.fdsll.cat | https://orcid.org/0000-0003-0210-7488
}

\begin{abstract}
Resumen: Introducción: El apoyo social percibido por la mujer tras el diagnóstico de cáncer de mama se relaciona con su calidad de vida y es predictor de la adaptación a la enfermedad. Objetivos: Comprender la evolución del apoyo social percibido por la mujer e identificar el apoyo social dos años después del diagnóstico de cáncer de mama. Métodos: Se diseñó un estudio cualitativo fenomenológico. La recogida de datos se realizó a través de entrevistas semi-estructuradas a 16 mujeres diagnosticadas de cáncer de mama en el 2016. Sobre las entrevistas se realizó un análisis de contenido temático entorno a las categorías principales del social (apoyo emocional, apoyo instrumental y apoyo informacional) asistido por el programa Nvivo v.12. Resultados: Del análisis de los datos emergieron 6 categorías temáticas: 1) Cuidadora y cuidada a lo largo del cáncer de mama, 2) Expectativas y satisfacción con el apoyo social recibido, 3) Necesidades y autocuidado durante el tratamiento, 4) Falta de apoyo instrumental: un problema estructural, 5) Información: problema o solución, 6) Falta de apoyo informacional como origen de problemas en el periodo de seguimiento. Conclusiones: El apoyo social recibido por la mujer con cáncer de mama disminuye con el tiempo y dos años después del diagnóstico se percibe una falta de apoyo sobre todo de tipo instrumental.
\end{abstract}

Palabras clave: Apoyo Social; Cáncer de Mama; Apoyo Estructural; Supervivencia; Investigación Cualitativa.

Perception of the Social Support Received after the Diagnosis of Breast Cancer: The Experience Two years after the Diagnosis.

Abstract: Introduction: The social support perceived by women after breast cancer diagnosis is related to their quality of life and is a predictor of adaptation to disease. Objectives: To understand the evolution of the social support perceived by the woman and to identify the social support two years after the diagnosis of breast cancer. Methods: A qualitative phenomenological study was used. The data collection was carried out through semistructured interviews with 16 women diagnosed with breast cancer in 2016. A thematic content analysis was carried out on the interviews around the main categories of social support (emotional support, instrumental support and informational support) assisted by the program Nvivo v.12. Results: From the analysis of the data, 6 themes emerged: 1) Caregiving and cared for throughout breast cancer, 2) Expectations and satisfaction with the social support received, 3) Needs and self-care during treatment, 4) Lack of instrumental support: a structura problem, 5) Information: problem or solution, 6) Lack of informational support as a source of problems in the follow-up period. Conclusions: The social support received by women with breast cancer decreases over time and two years after diagnosis a lack of support is perceived especially of an instrumental support.

Keywords: Social Support; Breast Cancer; Structural Support; Survivorship; Qualitative Research.

\section{Introducción}

El aumento en la prevalencia del cáncer de mama (CM) en los últimos años ha ido acompañado de una creciente preocupación acerca de los factores que mejoran el bienestar de la mujer y su calidad de vida (Badana et al., 2019; Kugbey et al., 2019). Diferentes estudios han apreciado como el apoyo social recibido por la mujer se ha relacionado con la calidad de vida tras el diagnóstico de CM (Yoo et al., 2010). 
El apoyo social cambia y evoluciona en base a las nuevas necesidades que experimenta la mujer en cada fase de la enfermedad (Drageset et al., 2012; Dumrongpanapakorn \& Liamputtong, 2017).

En el periodo del diagnóstico las reacciones de las mujeres son muy diversas y se identifican diferentes necesidades de apoyo (Mermer et al., 2016). Las mujeres entran en contacto con el sistema sanitario y se valora el apoyo informacional (Drageset et al., 2012). Posteriormente, durante el tratamiento, las mujeres destacan las consecuencias del tratamiento (náuseas, vómitos, pérdida del apetito, cansancio y pérdida de resistencia, etc.) y las necesidades que experimenta son de tipo instrumental (apoyo para la higiene, para cocinar, etc.), pero también de tipo emocional (sentirse apoyada, recibir ánimo) (Mermer et al., 2016; Smit et al., 2019). Por último, en el periodo de supervivencia conviven dos conductas ante la enfermedad: por un lado, la mujer se siente fuerte y optimista; por otro lado, la mujer experimenta miedo ante una posible recurrencia (Drageset et al., 2016).

Algunas investigaciones han apreciado como el apoyo social referido por las mujeres con CM tiende a disminuir con el tiempo (Fong et al., 2016). Esta disminución se acompaña de limitaciones sociales, es decir, de dificultades para expresar pensamientos y sentimientos relacionados con la enfermedad debido a las respuestas negativas de los demás (Tait \& Silver, 1989). En la etapa de seguimiento del CM, la falta de apoyo emocional y las limitaciones sociales supone un factor de riesgo para el desarrollo de ansiedad y depresión (Escalera et al., 2019; Puigpinós-Riera et al., 2018; Rivera Rivera \& Burris, 2019). Por ello nos planteamos responder la pregunta: ¿Cuál es la evolución del apoyo social percibido por la mujer dos años después del diagnóstico de CM?

En España, el apoyo social en mujeres diagnosticadas de CM ha sido un tema estudiado (Durá-Ferrandis et al., 2017; Puigpinós-Riera et al., 2018). Sin embargo, encontramos pocos estudios españoles que aborden el fenómeno desde una perspectiva cualitativa (Smit et al., 2019). Por esta razón, el estudio actual pretende comprender la evolución del apoyo social percibido por la mujer e identificar el apoyo social dos años después del diagnóstico de CM.

\section{Metodología}

\subsection{Diseño}

Este estudio se enmarca en un proyecto más amplio que aborda la experiencia de vivir CM dos años después del diagnóstico (Barcelona, España). Desde un paradigma interpretativo se utilizó un estudio cualitativo fenomenológico para comprender la experiencia de las mujeres en relación con el apoyo social percibido dos años después del diagnóstico de CM.

\subsection{Selección de Participantes}

Se realizó un muestreo intencional sobre la muestra de un estudio previo realizado en el mismo hospital (Estudio sobre el impacto socio-económico en mujeres diagnosticadas de cáncer de mama) (Masià et al., 2019). Se incluyeron a mujeres diagnosticadas de CM por primera vez en el año 2016 y con edades comprendidas entre los 50 y los 70 años. Se excluyó a aquellas mujeres con recidivas o en una etapa avanzada de la enfermedad. El número final de participantes se estableció en base a los principios de saturación de los datos.

A través de la enfermera de la unidad de mama del hospital se contactó telefónicamente con las mujeres que cumplían los criterios de inclusión. Una vez se les explicó el objetivo del estudio, aquellas que aceptaron participar y dieron su consentimiento recibieron una llamada de un miembro de equipo investigador para acordar día y hora para realizar la entrevista de forma presencial. 


\subsection{Recogida de Información}

Entre enero de 2018 y junio de 2019 se realizaron entrevistas semi-estructuradas basadas en el modelo de calidad de vida propuesto en el estudio de Ferrell et al (Ferrell et al., 1995). Dicho modelo estructuró las principales áreas del bienestar a valorar en la mujer en área física, psicológica, espiritual y social. Las preguntas se orientaron para comprender el apoyo social percibido en cada área y la satisfacción de la mujer a lo largo del tiempo. De tal manera encontramos preguntas del tipo ¿Cómo le ha afectado la enfermedad a nivel físico a lo largo de todo el proceso? ¿Cómo sintió que la apoyaron? Las entrevistas se realizaron por una enfermera del equipo con experiencia previa en dicha metodología. Estas se llevaron a cabo en una sala del hospital y fueron grabadas en audio.

\subsection{Análisis de los Datos}

Los audios de las entrevistas se transcribieron en su idioma original, castellano o catalán. Sobre las transcripciones se llevó a cabo un análisis de contenido temático con el soporte del programa Nvivo 12 (v.12). El proceso analítico se realizó por la investigadora principal y otra investigadora del equipo.

Se realizó una codificación mixta (cerrada y abierta). La codificación cerrada se realizó en torno a la categoría "Apoyo social" y el estudio de House (1981). Previamente a la codificación de las entrevistas se describieron los tipos de apoyo social que se identificarían en las entrevistas (apoyo emocional, apoyo estructural y apoyo informacional) (House, 1981). La codificación abierta se llevó a cabo paralelamente, y a medida que se iban leyendo las entrevistas emergieron nuevas categorías (fuente de apoyo, falta de apoyo, afrontamiento, fases de la enfermedad, etc).

Una vez las entrevistas estuvieron codificadas se realizó la exploración de la información a través de una consulta de grupo y una matriz de codificación mediante Nvivo 12. Esta función permitió profundizar en las relaciones entre las diferentes categorías.

Finalmente, la validación de los resultados del análisis se realizó mediante la triangulación de la información con la enfermera de la unidad de mama del hospital.

\subsection{Aspectos Éticos}

El estudio fue aprobado por el Comité de Ética de la Investigación Clínica (CEIC) del hospital (IIBSP-CAM-2016-63). Todas las mujeres firmaron el consentimiento informado previa participación en el estudio. Con el objetivo de mantener el anonimato de las participantes se utilizó un pseudónimo que garantizó la no identificación de las entrevistadas.

\section{Resultados}

\subsection{Características de las Participantes}

Se realizaron un total de 16 entrevistas a mujeres diagnosticadas de CM en 2016. La media de edad de las mujeres entrevistadas fue de 60 años en el diagnóstico. El 100\% de las mujeres fueron intervenidas de tumorectomía. En cuanto a los tratamientos recibidos, el $50 \%$ de las entrevistadas recibieron radioterapia, quimioterapia y hormonoterapia, y el $37 \%$ recibieron radioterapia y hormonoterapia. Como excepción, una mujer recibió radioterapia y quimioterapia, y otra mujer recibió únicamente hormonoterapia. A nivel socioeconómico el $25 \%$ de las entrevistadas tenían ingresos bajos, el $44 \%$ ingresos medios y el $31 \%$ ingresos altos. 


\subsection{Temas emergentes durante el análisis}

Del análisis emergieron seis temas en base a las categorías principales (Tabla 1).

Tabla 1: Temas revelados en el análisis.

\begin{tabular}{ccl}
$\begin{array}{c}\text { APOYO } \\
\text { EMOCIONAL }\end{array}$ & 1. & $\begin{array}{l}\text { Cuidadora y cuidada a lo largo del cáncer } \\
\text { de mama }\end{array}$ \\
$\begin{array}{c}\text { APOYO } \\
\text { INSTRUMENTAL }\end{array}$ & 2. & $\begin{array}{l}\text { Expectativas y satisfacción con el apoyo } \\
\text { recibido }\end{array}$ \\
3. & $\begin{array}{l}\text { Necesidades y autocuidado durante el } \\
\text { tratamiento }\end{array}$ \\
APOYO INFORMACIONAL & 4. & $\begin{array}{l}\text { Falta de apoyo instrumental: un problema } \\
\text { estructural }\end{array}$ \\
& 5. & Información: problema o solución \\
\hline
\end{tabular}

\subsubsection{Cuidadora y Cuidada a lo Largo del Cáncer de Mama}

El diagnóstico conlleva necesidades relacionadas con la enfermedad, y otras relacionadas con el rol de la mujer dentro de su familia. En el momento del diagnóstico sus principales preocupaciones giran en torno al pronóstico de la enfermedad, pero también les preocupa saber cómo comunicar la enfermedad y el efecto de la noticia en la familia. Una parte de las mujeres no recibió apoyo al no poder comentar sus preocupaciones a la familia (con padres muy mayores o hijos centrados en el trabajo y la crianza).

Yo en casa no lo podía decir porque tengo una madre de 90 años ... Claro todo eso te afecta, que tú en casa no puedas llorar, no puedas explicárselo a una madre... (Neus, 60 años)

Durante el tratamiento las mujeres refirieron necesitar mucho apoyo emocional. Sin embargo, también encontramos algún caso en que la mujer no necesitó ese apoyo debido a su larga experiencia como cuidadora principal.

Por último, dos años después del diagnóstico, las mujeres hacen referencia a la falta de apoyo emocional sobre todo para verbalizar el miedo. En general se percibe como la familia y el entorno más próximo necesita distanciarse de la enfermedad y no hablar del tema.

A veces le quitan tanta importancia... Y yo necesito decirlo, necesito expresar lo que siento...Y a veces no me dejan. (Neus, 60 años)

\subsubsection{Expectativas y Satisfacción con el Apoyo Recibido.}

La satisfacción de las mujeres entrevistadas se basa en sus expectativas de apoyo. Las mujeres utilizan expresiones como "actuaron como esperaba" o "han estado ahí" para hacer referencia al apoyo satisfactorio. Sin embargo, también se identifican expresiones como "no lo que yo habría querido", "me gustaría no tenerlo que decir" o "me decepcionó" que hacen referencia a una falta de apoyo en relación con las expectativas de la mujer.

Me decepcionó un poco mi marido. Es la forma de ser de cada uno, pero en esos momentos más flojos puede que sí que me encontrara como más sola. (Lola, 66 años). 


\subsubsection{Necesidades y autocuidado durante el tratamiento.}

El apoyo instrumental se valora más durante el periodo de tratamiento. En ese momento las mujeres experimentan más limitaciones físicas, que les impiden realizar las actividades básicas de la vida diaria.

Gracias a mi marido que ha estado un año pendiente de mí en todo y por todo, yendo a comprar, haciendo las cosas, hacer la comida, ducharme, lavarme, o sea todo, todo, (Inés, 70 años).

\subsubsection{Falta de apoyo instrumental: un problema estructural.}

En el periodo de seguimiento (dos años después del diagnóstico) es donde se identifica una disminución de todos los tipos de apoyo, y paralelamente un aumento de la falta de apoyo sobre todo instrumental. Esta falta de atención se atribuye al hospital, y en general se percibe que las consecuencias se tratan por separado, por diferentes especialistas y que no se consigue una solución satisfactoria. Algunas mujeres buscan atención privada para satisfacer sus necesidades.

Encontramos mujeres en el estudio que refieren dificultad para reincorporarse al ámbito laboral. Dificultades para encontrar trabajo, problemas con el trabajo que desarrollaban antes de la enfermedad, etc.

Cuando me incorporé hubieron varios despidos y aquello me afectó. Me dieron tareas más físicas, cosa que he llevado muy mal y llegó un punto que tuve un síncope. (Sofía, 55 años).

Por último, las mujeres más mayores de estudio presentan menos redes de apoyo. En los discursos las mujeres asumen el aislamiento como parte del proceso del envejecimiento, y no identifican la soledad como un problema que agrava las consecuencias de haber padecido el CM.

\subsubsection{Información: problema o solución}

El apoyo informacional se atribuyó principalmente a profesionales sanitarios del hospital y a mujeres que habían pasado la enfermedad. Para algunas mujeres recibir información las capacitó en la toma de decisiones y les proporcionó cierto control sobre la situación. Sin embargo, para otras mujeres la información, sobre todo en momentos iniciales, les produjo ansiedad.

Me sentí muy apoyada en todo. Todo lo que preguntabas en el momento una respuesta... (Neus, 60 años).

\subsubsection{Falta de apoyo informacional como origen de problemas en el periodo de seguimiento.}

Las mujeres entrevistadas por lo general están satisfechas con la información recibida por parte de los profesionales del hospital en el momento del diagnóstico y del tratamiento. Es en la fase de seguimiento donde refieren una falta de información para abordar las consecuencias del tratamiento, sobre las posibles complicaciones a largo plazo, etc. Esto genera ansiedad cuando la mujer presenta síntomas o dolencias dos años después.

Tuve un susto porque me noté un bultito. Me hicieron pruebas y era agua que se había generado de la radioterapia. (Neus, 60 años).

Yo ya sé que no tiene remedio, pero no sé a lo mejor me podrían decir que haga algún ejercicio, alguna cosa para estar mejor... (Marina, 56 años). 


\section{Discusión}

Acorde a estudios previos (Fong et al., 2016) la disminución del apoyo social está relacionada con las necesidades y expectativas que las mujeres experimentan según la fase de la enfermedad (Den Oudsten et al., 2010).

El apoyo emocional es el tipo de apoyo que más se valora a lo largo de todo el proceso de la enfermedad. Este ofrece a la mujer motivación y energía para afrontar la incertidumbre del diagnóstico y el tratamiento (Dumrongpanapakorn \& Liamputtong, 2017). Sin embargo, se experimenta una falta de apoyo al anteponer el cuidado hacia los demás, y ante dificultades para expresar sus necesidades al entorno (Coyne et al., 2012). Además, dos años después del diagnóstico se aprecian limitaciones sociales, lo cual impide a la mujer expresar su miedo ante una posible recurrencia (Drageset et al., 2020; Rivera Rivera \& Burris, 2019).

El apoyo instrumental se considera fundamental durante el periodo del tratamiento, cuando las mujeres experimentan más dificultades para satisfacer su propio autocuidado. Dos años después del diagnóstico, las mujeres destacan una falta de apoyo instrumental que atribuyen sobre todo al hospital y al trabajo. Una vez finalizado el tratamiento las mujeres no sienten una continuidad en su atención. Este hecho lleva a la mujer con más recursos a buscar alternativas y satisfacer sus necesidades de forma privada (Sousa Rodrigues Guedes et al., 2020). A su vez, las mujeres describen cambios a nivel laboral como consecuencia de la enfermedad (Bilodeau et al., 2019). El abordaje de dichos cambios supone un reto para la mujer sobre todo cuando desarrolla trabajos físicos (Paul et al., 2016; Vanderpool et al., 2013). Por último, las mujeres más mayores del estudio deben hacer frente a las consecuencias de la enfermedad en el contexto propio del envejecimiento (Angarita et al., 2020). Por tanto, los resultados muestran como la falta de apoyo instrumental en la fase de seguimiento favorece las desigualdades sociales y las mujeres con menos ingresos y más edad serán más susceptible de no poder atender las consecuencias de la enfermedad (Dialla et al., 2012; Puigpinós-Riera et al., 2018).

Por último, el apoyo informacional se percibe de forma diferente por las mujeres del estudio. Algunas mujeres necesitan información sobre la enfermedad, y conocer los detalles del proceso les aporta cierto control sobre la situación (Aunan et al., 2019; Drageset et al., 2020; Faller, Brahler, et al., 2017). Sin embargo, para otras mujeres la información genera ansiedad, y prefieren vivir el proceso paso a paso (Faller, Strahl, et al., 2017). En la fase de seguimiento, las mujeres perciben una falta de información acerca de las secuelas y los cambios físicos (Vachon, 2006). Como consecuencia la mujer puede llegar a confundir algunos síntomas propios de la enfermedad o el tratamiento con un nuevo proceso canceroso (Aunan et al., 2019; Smit et al., 2019).

La metodología cualitativa ofrece resultados enriquecedores a partir del análisis de los discursos de las mujeres diagnosticadas de CM. Los resultados no solo nos permiten conocer el apoyo social percibido por la mujer, sino que también orienta sobre los motivos por los que un apoyo no resulta satisfactorio y sobre las necesidades insatisfechas dos años después del diagnóstico. Nuestros hallazgos ofrecen información relevante para la mejora de la asistencia (sanitaria, laboral y social) de las mujeres con CM en el periodo de seguimiento.

La limitación principal del estudio actual se basa en el riesgo de sesgo de memoria. Al tratarse de un estudio retrospectivo sobre el apoyo social percibido dos años después del diagnóstico existe el riesgo de que el recuerdo sobre ese periodo esté alterado.

\section{Conclusiones}

El apoyo social que recibe la mujer cambia y evoluciona durante los dos años posteriores al diagnóstico de CM. El apoyo emocional es el apoyo que más se valora a lo largo del tiempo, sin embargo, con el paso de los años tiende a disminuir. Esta disminución tiene consecuencias sobre el bienestar de la mujer, la cual no encuentra comprensión para expresar sus miedos y preocupaciones. 
El apoyo instrumental, se valora sobre todo en la etapa de tratamiento, cuando la mujer experimenta más limitaciones físicas. Con el paso del tiempo también disminuye, y una falta de compromiso por parte de las autoridades sanitarias puede aumentar las desigualdades sociales, donde las mujeres con menos recursos y más edad tienen más riesgo de no atender las consecuencias de la enfermedad. Por último, el apoyo informacional se puede valorar de forma positiva o negativa en función de las necesidades de la mujer. La falta de información a lo largo del tiempo puede originar problemas en el periodo de seguimiento. Comprender la evolución del apoyo social percibido por la mujer con CM es necesario para abordar las consecuencias a largo plazo y satisfacer las necesidades de una enfermedad de gran prevalencia en la sociedad española.

\section{Agradecimientos}

Las autoras queremos agradecer la contribución en el artículo de Magda Ciendones, enfermera de la Unidad de Mama del hospital de la Santa Creu y Sant Pau.

\section{Referencias}

Angarita, F. A., Zhang, Y., Elmi, M., \& Look Hong, N. J. (2020). Older women's experience with breast cancer treatment: A systematic review of qualitative literature. Breast, 54, 293-302. https://doi.org/10.1016/j.breast.2020.11.009

Aunan, S. T., Wallgren, G. C., \& Sætre Hansen, B. (2019). Breast cancer survivors' experiences of dealing with information during and after adjuvant treatment: A qualitative study. Journal of Clinical Nursing, 28(15-16), 3012-3020. https://doi.org/10.1111/jocn.14700

Badana, A. N. S., Marino, V. R., Templeman, M. E., McMillan, S. C., Tofthagen, C. S., Small, B. J., \& Haley, W. E. (2019). Understanding the roles of patient symptoms and subjective appraisals in well-being among breast cancer patients. Supportive Care in Cancer, 27(11), 4245-4252. https://doi.org/10.1007/s00520-019-04707-2

Bilodeau, K., Tremblay, D., \& Durand, M.-J. (2019). Return to work after breast cancer treatments: Rebuilding everything despite feeling "in-between". European Journal of Oncology Nursing, 41, 165-172. https://doi.org/10.1016/j.ejon.2019.06.004

Coyne, E., Wollin, J., \& Creedy, D. K. (2012). Exploration of the family's role and strengths after a young woman is diagnosed with breast cancer: Views of women and their families. European Journal of Oncology Nursing, 16(2), 124-130. https://doi.org/10.1016/j.ejon.2011.04.013

Den Oudsten, B. L., Van Heck, G. L., Van der Steeg, A. F. W., Roukema, J. A., \& De Vries, J. (2010). Personality predicts perceived availability of social support and satisfaction with social support in women with early stage breast cancer. Supportive Care in Cancer, 18(4), 499-508. https://doi.org/10.1007/s00520-009-0714-3

Dialla, P. O., Dabakuyo, T. S., Marilier, S., Gentil, J., Roignot, P., Darut-Jouve, A., Poillot, M. L., Quipourt, V., \& Arveux, P. (2012). Population-based study of breast cancer in older women: prognostic factors of relative survival and predictors of treatment. BMC Cancer, 12. https://doi.org/10.1186/1471-2407-12-472

Drageset, S., Lindstrøm, T. C., \& Ellingsen, S. (2020). "I Have Both Lost and Gained." Norwegian Survivors' Experiences of Coping 9 Years after Primary Breast Cancer Surgery. Cancer Nursing, 43(1), E30-E37. https://doi.org/10.1097/NCC.0000000000000656

Drageset, S., Lindstrøm, T. C., Giske, T., \& Underlid, K. (2012). “The support i need”: Women's experiences of social support after having received breast cancer diagnosis and awaiting surgery. Cancer Nursing, 35(6), 39-47. https://doi.org/10.1097/NCC.0b013e31823634aa

Drageset, S., Lindstrøm, T. C., \& Underlid, K. (2016). "I just have to move on”: Women's coping experiences and reflections following their first year after primary breast cancer surgery. European Journal of Oncology Nursing, 21, 205-211. https://doi.org/10.1016/j.ejon.2015.10.005 
Dumrongpanapakorn, P., \& Liamputtong, P. (2017). Social support and coping means: the lived experiences of Northeastern Thai women with breast cancer. Health Promotion International, 32(5), 768-777. https://doi.org/10.1093/heapro/dav023

Durá-Ferrandis, E., Mandelblatt, J. S., Clapp, J., Luta, G., Faul, L. A., Kimmick, G., Cohen, H. J., Yung, R. L., \& Hurria, A. (2017). Personality, coping, and social support as predictors of longterm quality-of-life trajectories in older breast cancer survivors: CALGB protocol 369901 (Alliance). Psycho-Oncology, 26(11), 1914-1921. https://doi.org/10.1002/pon.4404

Escalera, C., Santoyo-Olsson, J., Stewart, A. L., Ortiz, C., \& Nápoles, A. M. (2019). Social support as a moderator in the relationship between intrusive thoughts and anxiety among Spanishspeaking Latinas with breast cancer. Psycho-Oncology, 28(9), 1819-1828. https://doi.org/10.1002/pon.5154

Faller, H., Brahler, E., Harter, M., Keller, M., Schulz, H., Wegscheider, K., Weis, J., Boehncke, A., Reuter, K., Richard, M., Sehner, S., Koch, U., \& Mehnert, A. (2017). Unmet needs for information and psychosocial support in relation to quality of life and emotional distress: A comparison between gynecological and breast cancer patients. Patient Education and Counseling, 100(10), 1934-1942. https://doi.org/10.1016/j.pec.2017.05.031

Faller, H., Strahl, A., Richard, M., Niehues, C., \& Meng, K. (2017). The prospective relationship between satisfaction with information and symptoms of depression and anxiety in breast cancer: A structural equation modeling analysis. Psycho-Oncology, 26(11), 1741-1748. https://doi.org/10.1002/pon.4358

Ferrell, A. B. R., Dow, K. H., \& Grant, M. (1995). Measurement of the Quality of Life in Cancer Survivors. Springer, 4, 523-531.

Fong, A. J., Scarapicchia, T. M. F., Mcdonough, M. H., Wrosch, C., \& Sabiston, C. M. (2016) Changes in social support predict emotional well-being in breast cancer survivors. Psy, 26(5), 664-671.

House, J. S. (1981). Work Stress and Social Support. California, Addison We. http://mendeley.csuc.cat/fitxers/86193f5fd1ec22acb2bade10d6b1840a

Kugbey, N., Meyer-Weitz, A., \& Oppong Asante, K. (2019). Mental adjustment to cancer and quality of life among women living with breast cancer in Ghana. International Journal of Psychiatry in Medicine, 54(3), 217-230. https://doi.org/10.1177/0091217418805087

Masià, J., Merchán-Galvis, Salas, K., Requeijo, C., Cánovas, E., Quintana, M. J., Bonfill, X., Ayala, J. M., Cosp, X. B., Ruiz, M. J. Q., Lorenzo, C. R., Gama, K. S., Martínez, E. C., Molins, A. B., Vilanova, B. G. V., Duarte, A. M., Playa, G. P., Verdum, J. I., Raspall, M. J. F., ... Galvis, Á. M. (2019). Socio-economic impact on women diagnosed and treated for breast cancer: a cross-sectional study. Clinical and Translational Oncology, 21(12), 1736-1745. https://doi.org/10.1007/s12094-019-02185-w

Mermer, G., Nazli, A., \& Ceber, E. (2016). Social perceptions of breast cancer by women still undergoing or having completed therapy: A qualitative study. Asian Pacific Journal of Cancer Prevention, 17(2), 503-510. https://doi.org/10.7314/APJCP.2016.17.2.503

Paul, C., Boyes, A., Hall, A., Bisquera, A., Miller, A., \& O’Brien, L. (2016). The impact of cancer diagnosis and treatment on employment, income, treatment decisions and financial assistance and their relationship to socioeconomic and disease factors. Supportive Care in Cancer, 24(11), 4739-4746. https://doi.org/10.1007/s00520-016-3323-y

Puigpinós-Riera, R., Graells-Sans, A., Serral, G., Continente, X., Bargalló, X., Domènech, M., Espinosa-Bravo, M., Grau, J., Macià, F., Manzanera, R., Pla, M., Quintana, M. J., Sala, M., \& Vidal, E. (2018). Anxiety and depression in women with breast cancer: Social and clinical determinants and influence of the social network and social support (DAMA cohort). Cancer Epidemiology, 55, 123-129. https://doi.org/10.1016/j.canep.2018.06.002

Rivera Rivera, J. N., \& Burris, J. L. (2019). A Systematic Literature Review and Head-to-Head Comparison of Social Support and Social Constraint in Relation to the Psychological Functioning of Cancer Survivors. Annals of Behavioral Medicine, 54(3), 176-192. https://doi.org/10.1093/abm/kaz037

Smit, A., Coetzee, B. J. sean, Roomaney, R., Bradshaw, M., \& Swartz, L. (2019). Women's stories of living with breast cancer: A systematic review and meta-synthesis of qualitative evidence. Social Science and Medicine, 222(December 2018), 231-245. https://doi.org/10.1016/j.socscimed.2019.01.020 
Vol. 8 | Investigación Cualitativa en Salud: Avances y Desafíos

Sousa Rodrigues Guedes, T., Patrocínio da Silva Barros, C., Dantas de Oliveira, N. P., Martins Holanda, A., Albuquerque Reis, M., Rocha e Silva, B. L., Barbosa Otoni Gonçalves Guedes, M., Jerez-Roig, J., \& Bezerra de Souza, D. L. (2020). Social support in the healthcare of women submitted to breast cancer treatment. Women and Health, 60(8), 899-911. https://doi.org/10.1080/03630242.2020.1767263

Tait, R., \& Silver, R. C. (1989). Coming to terms with major negative life events (J. S. Uleman \& J. A. Bargh (Eds.) (ed.); Unintended). Guilford Press.

Vachon, M. (2006). Psychosocial Distress and Coping After Cancer Treatment. Cancer Nursing, 29(Supplement), 26-31. https://doi.org/10.1097/00002820-200603002-00011

Vanderpool, R. C., Swanberg, J. E., \& Chambers, M. D. (2013). A Narrative Review of the Confluence of Breast Cancer and Low-wage Employment and Its Impact on Receipt of Guideline-recommended Treatment. GLOBAL ADVANCES IN HEALTH AND MEDICINE, 2(5), 75-85. https://doi.org/10.7453/gahmj.2013.046

Yoo, G. J., Levine, E. G., Aviv, C., Ewing, C., \& Au, A. (2010). Older women, breast cancer, and social support. Supportive Care in Cancer, 18(12), 1521-1530. https://doi.org/10.1007/s00520-009-0774-4 